Relations industrielles

Industrial Relations

\title{
La grève de l'amiante
}

\section{Alfred Charpentier}

Volume 19, numéro 2, avril 1964

URI : https://id.erudit.org/iderudit/1021312ar

DOI : https://doi.org/10.7202/1021312ar

Aller au sommaire du numéro

\section{Éditeur(s)}

Département des relations industrielles de l’Université Laval

ISSN

0034-379X (imprimé)

1703-8138 (numérique)

Découvrir la revue

\section{Citer cet article}

Charpentier, A. (1964). La grève de l'amiante. Relations industrielles / Industrial Relations, 19(2), 217-238. https://doi.org/10.7202/1021312ar
Résumé de l'article

Dans le présent article, l'auteur fait une analyse complète des dossiers de la Commission des relations ouvrières portant les numéros 2158, 2276, 2296 et 2116, relatifs à la grève de l'amiante survenue en 1949 à Asbestos, à Thetford-les-Mines et autres endroits de la région.
Tous droits réservés ( Département des relations industrielles de l’Université Laval, 1964
Ce document est protégé par la loi sur le droit d'auteur. L’utilisation des services d'Érudit (y compris la reproduction) est assujettie à sa politique d'utilisation que vous pouvez consulter en ligne.

https://apropos.erudit.org/fr/usagers/politique-dutilisation/ 


\title{
La grève de l'amiante: version nouvelle
}

\author{
Alfred Charpentier
}

Dans le présent article, lauteur fait une analyse complète des dossiers de la Commission des relations ouvrières portant les numéros $2158,2276,2296$ et 2116, relatifs d la grève de lamiante survenue en 1949 d Asbestos, d̀ Thetford-les-Mines et autres endroits de la région.

Déclarée le 14 février 1949 à la mine, au moulin et à la fabrique de la Canadian Johns-Manville Co. d'Asbestos, la grève se propagea le lendemain aux quatre mines de l'Asbestos Corporation, à ThetfordMines: King, Vimy-Ridge, Beaver et British Canadian. En de ça d'une semaine la grève s'étendit aux trois autres compagnies minières de la région: Quebec Asbestos Corporation (East-Broughton), Johnson's Co. Ltd (Black Lake), Flintkote Mines Ltd (Thetford-Mines). La grève dura quatre mois et demi à Asbestos et Thetford-Mines, une semaine environ dans les deux autres endroits.

Le rôle de la Commission de relations ouvrières sera particulièrement révélé par l'analyse du dossier 2158 se rapportant au différend entre Canadian Johns-Manville Co. Ltd. et le Syndicat national catholique de l'Amiante d'Asbestos Inc., mais les faits et renseignements émanant de ce dossier seront complétés, au besoin, par des références à l'un ou l'autre des trois autres dossiers $(2116,2206$ et 2277 ) se rapportant aux autres compagnies minières précitées.

\section{ARRTÈrRe PLAN}

\section{Fondation des syndicats}

La fondation des syndicats de mineurs de l'amiante dans les différents endroits de la région concernée eut lieu à Thetford-Mines et à Asbestos en 1935, - East-Broughton en 1936, ${ }^{1}$ à Black Lake en 1944 et, en 1946, un second syndicat fut formé à Thetford-Mines chez les employés de la Flintkote Mines. Les cinq syndicats furent reconnus légalement par la Commission de relations ouvrières à la fin de l'année 1947.

(1) Ces deux syndicats furent organisés par l'auteur de cet article. 
Situation du syndicat d Asbestos de 1946 d 1948

Pendant une dizaine d'années avant sa certification en 1947, le Syndicat national catholique de l'Amiante, d'Asbestos, avait entretenu des rapports réguliers sinon toujours harmonieux avec la Canadian Johns-Manville. Jusqu'au début de 1944, il vécut sous le régime des ententes de gré à gré avec la compagnie, et ensuite syndicat et compagnie furent régis par le décret fédéral C.P. 1003 jusqu'à son abolition, le 31 août 1948.

Depuis novembre 1945, la Fédération nationale catholique des employés de l'Amiante était accréditée comme mandataire par le Conseil national du Travail pour deux de ses syndicats affiliés: celui des employés de la Canadian Johns-Manville et celui des employés de l'Asbestos Corporation Ltd.

Or, il appert que c'est le ler février 1946 que le «Syndicat »et la \& Johns-Manville » conclurent leur dernière convention collective sous l'autorité du décret et sous la surveillance du Conseil régional du Travail de Québec. Le 18 avril le Conseil national du Travail accorda, pour deux ans, toutes les améliorations demandées conjointement par les deux parties. (V. Gazette du Travail, 1946, pp. 631, 632 et 818). La convention en cours expirait le 31 janvier 1948. Le 29 mai 1946, le Syndicat déposa la convention au ministère provincial du Travail. Le 5 décembre suivant la Commission de relations ouvrières est informé du dépôt de la convention au ministère du Travail. (Ces faits indiquent que le décret fédéral C.P. 1003 avait cessé en pratique d'être appliqué dans la province de Québec depuis la fin de 1946; le Conseil régional cessa d'exister en mars 1947.

C'est au mois d'août 1947 que le Syndicat demande à la C.R.O. de lui émettre un certificat de reconnaissance syndicale. La Commission lui répond que la convention en vigueur n'expirant que le 31 janvier 1948, elle ne pourra considérer sa demande que dans le délai prévu à l'article $16^{2}$ de la Loi des relations ouvrières. Mais le même jour (19 août) que la Commission apprend au Syndicat que sa demande est prématurée, elle reçoit de ce dernier une demande, datée de la veille, de suspendre sa requête en reconnaissance syndicale. Le même jour Jean Marchand, directeur de l'organisation de la C.T.C.C., télégraphie le même message à la C.R.O.

Ce n'est que deux mois plus tard, le 27 octobre, que la Commission est requise par le Syndicat de procéder avec la requête en reconnaissance. Effectivement, le 23 de ce mois, la Commission avisera la Canadian Johns-Manville de la réception de la requête syndicale et

(2) L'article 16 de la loi de relations ouvrières stipule qu'une telle requête doit être présentée à la C.R.O. entre le 60ème et le 30 ème jour précédant lexpiration d'une convention collective. 
lui demandera de faire des représentations dans un délai de sept jours de la date de la requête du Syndicat le 13 août. Ce même jour, le procureur de la compagnie avait répondu que \&ses clients renoncent à tout délai » et désirent la certification immédiate du Syndicat national de l'Amiante, d'Asbestos.

Les syndicats demandent de certifier la fédération

Comment expliquer ici que le Syndicat ait attendu jusqu'au 27 octobre pour écrire à la C.R.O. et lui demander de procéder à l'étude de sa requête, tenue en suspens depuis la mi-août? Une seule explication est plausible, c'est que cette lettre du Syndicat, datée le 27 octobre, ne sert qu'à confirmer une demande verbale présumément faite quelques jours précédemment à un membre de la Commission par un responsable du Syndicat ${ }^{3}$. Mais il importe de remarquer dans la lettre de ce dernier, qu'il prie la C.R.O. de modifier sa requête en reconnaissance de façon à ce que le certificat de reconnaissance syndicale soit émis au nom de la Fédération nationale des employés de l'Industrie minière plutôt qu'à lui-même, afin que ses droits d'agents négociateurs soient exercés par la «Fédération». Celle-ci informe la C.R.O. le lendemain qu'elle consent à être « certifiée en lieu et place » du Syndicat.

\section{Fédération reconnue comme mandatatre}

Deux jours après cette décision de la Commission, la Fédération lui demande, le 31 octobre, d'être «accréditée » pour quatre autres syndicats, vis-à-vis quatre autres compagnies, à savoir: une à EastBroughton, la Quebec Asbestos Corporation et trois à Thetford-Mines: Asbestos Corporation Ltd, Flintkote Mines Inc. Ltd, Johnson's Co. Ltd. Malgré la demande maladroite de la Fédération d'être «certifiée en lieu et place $\gg$ de ses syndicats - ce que la loi ne permettait pas la Commission accepta néanmoins la Fédération comme agent négociateur de ses syndicats, en même temps qu'elle émettait à chacun de ces derniers, un certificat de reconnaissance syndicale. Ce qui eut lieu le 3 décembre 1947.

\section{LES FAITS}

Négociations d Thetford-Mines

Depuis le début de décembre, des négociations étaient en cours entre trois autres syndicats de la Fédération pour renouveler leurs conventions collectives avec l'Asbestos Corporation Ltd, avec la Johnson's Co. Ltd, et avec la Flintkote Mines Ltd, située chacune à Thetford-Mines.

(3) A l'époque, pareille procédure officieuse était fréquente entre la C.R.O. et les organisateurs syndicaux. 
Le 6 janvier 1948, les mineurs de deux compagnies déclarent la grève, sauf à la Flintkote Mines, cette compagnie s'étant engagée par écrit de se conformer aux conditions qu'accepteraient les deux autres compagnies au stade de la conciliation ou de l'arbitrage. Un conciliateur du ministère du Travail dépêché auprès des parties, les 12 et 14 janvier, pour tenter la conciliation, rapporte: 1 - que la grève a duré trois jours; 2 - que la lenteur des négociations en est la cause conjointement avec la demande des mineurs des trois compagnies de Thetford-Mines de conserver le différentiel de 2 cents l'heure de plus que n'obtiennent les mineurs employés à la Vimy Ridge Mine, située à quelque vingt milles au sud de Thetford-Mines.

Le conciliateur Noël Bérubé effectua une entente et termina son rapport en déclarant qu'une convention collective allait être signée dans quelques jours entre toutes les parties concernées dans les entreprises minières précitées.

\section{Différent d la Canadian Johns-Manville}

Au début de février 1948 surgit un différend entre le syndicat des mineurs d'Asbestos et la Canadian Johns-Manville Co. Ltd. Le 9 février, de Shawinigan Falls, Jean Marchand adresse un télégramme à la C.R.O. lui demandant l'intervention du Service de conciliation pour régler un grief touchant la convention collective. Peu après, la Commission ayant demandé quelques détails sur le grief en cause, Marchand précise ces griefs dans une lettre en date du 19 février. Il s'agit de la suppression d'un employé dans une équipe de quatre hommes, et ce, sans avoir consulté le syndicat, ce qui serait contraire à la convention. Le lendemain, la Commission répond à Jean Marchand que sa demande est référée au ministère du Travail, vu qu'il s'agit d'un gruef qui relève de la Loi des différends ouvriers. Le même jour la Commission communique la documentation requise au ministère du Travail avec prière d'y donner suite. Accusé de réception est faite par ce dernier le 23 février, disant que le Service de conciliation a instruction d'intervenir.

\section{Seconde demande de conciliation}

Un mois et demi s'écoule sans intervention conciliatrice. Le 16 avril, par son «représentant » André Roy (secrétaire de la C.T.C.C.) le Syndicat réitère sa demande à la C.R.O. pour un conciliateur; il ne rappelle pas sa demande antérieure (lettre de J. Marchand). La Commission l'informe encore que le différend relève de la Loi des différends ouvriers dont l'application dépend du ministère du Travail et que sa correspondance est référée à ce dernier. Lequel réfère aussitôt la susdite demande au Service de conciliation en lui demandant de «faire le nécessaire 》.

Le syndicat demande à la C.R.O. l'intervention d'un conciliateur. La requête est signée par $M$. Jean Marchand, organisateur en chef de la C.T.C.C. La Commission informe le syndicat que la requête devra 
être transmise au ministère du Travail vu que le grief relève de la Loi des différends ouvriers, mais, avant de ce faire, elle lui demande des précisions sur le différend - ce qui n'était pas nécessaire en l'occurence. Et ce n'est qu'après avoir obtenu ces renseignements que la Commission envoie la requête du Syndicat au Service de conciliation du ministère du Travail. Aucun conciliateur ne s'étant présenté sur les lieux après un mois et demi d'attente, le syndicat réitère sa demande à la C.R.O. Elle est signée cette fois-ci par M. André Roy, secrétaire général de la C.T.C.C. Ignore-t-il l'envoi de la première requête? Son geste semble l'indiquer. Du reste il n'en parle pas. Pas plus ne se plaint-il du mois et demi d'attente. La C.R.O. lui répond comme elle l'a fait à Jean Marchand, que sa requête est référée au ministère du Travail. Les deux dirigeants de la C.T.C.C. paraissent ignorer que l'on ne doit s'adresser à la C.R.O. pour obtenir l'intervention conciliatrice qu'à l'occasion de l'échec de négociations pour la conclusion d'une convention ou de son renouvellement. C'est en pareil cas que la Commission a besoin de connaître les points de désaccord afin que le conciliateur, informé en conséquence, puisse mieux savoir comment absorder les parties en cause.

Le conciliateur, Georges Lépine, n'arriva à Asbestos que le 27 avril. Les erreurs de procédures commises par les deux dirigeants de la C.T.C.C. sont peut-être partiellement responsables du retard de l'intervention conciliatrice dans le cas présent, mais il reste inexplicable que la première requête du syndicat en date du 9 février, transmise au ministère du Travail une douzaine de jours après, ait été laissée sans suite aucune, ayant nécessité une seconde requête du syndicat le 16 avril.

M. Georges Lépine n'arriva que de justesse, le 27 avril, pour éviter la grève qui devait éclater le lendemain dans deux départements de la mine de la Canadian Johns-Manville, en obtenant de la compagnie de suspendre le changement de tâche ordonné pour le lendemain. Les parties convinrent d'assister à une séance de conciliation, le 10 mai.

\section{Changement du conciliateur}

Fait singulier, deux jours avant la soumission de ce rapport au ministre du Travail, celui-ci désigne un autre conciliateur pour continuer le travail de M. Lépine. Il s'agit de M. Léopold Rogers. Il est curieux que M. Lépine n'ait fait qu'amorcer la conciliation.

Dans son rapport incomplet soumis le 29 au ministre du Travail, il disait avoir procédé en vertu de l'article 13 de la Loi des relations ouvrières. Avait-il reçu instruction de procéder de cette façon, ou a-t-il agi ainsi de son chef? De toute façon c'était une grave erreur. Son successeur, Léopold Rogers, dira lui, avoir concilié en regard de l'article 9 de la Loi des différends ouvriers. Ce qui répondait à la situation. Après quelques entrevues particulières avec les représentants de chaque partie, il tient, le 13 mai, une séance de conciliation vouée à 
l'échec. Les parties consentirent à faire trancher leur différend par un conseil d'arbitrage.

\section{Conseil d'arbitrage}

Informé de l'échec de la conciliation, le chef du Service de conciliation, M. Cyprien Miron, demande, le 21 mai, au Syndicat et « JohnsManville » de se choisir un arbitre pour former un conseil d'arbitrage. Constitué du Juge Poisson comme président, de Me André Sabourin pour la compagnie et de M. Daniel Lessard pour le Syndicat, le dit conseil ne fait son rapport qu'en date du 19 octobre. Et la décision ne sera que majoritaire, le représentant syndical étant dissident. La décision majoritaire rejette la demande du Syndicat, jugeant «qu'à tous égards, il n'y a eu aucun changement dans les conditions ou méthodes de travail ». Dans son rapport minoritaire, le représentant du Syndicat maintient que la tâche de travail a été modifiée, justifiant une augmentation du taux de salaire, ou que, du moins, le conseil aurait pu exiger le maintien des conditions de travail existant antérieurement. D’autre part le président et l'arbitre patronal ont prétendu qu'il n'a pas été prouvé qu'une clause quelconque de la convention avait été enfreinte. Ils disent que s'il y a eu un employé de lésé ce serait en raison d'un « effort physique imperceptiblement plus considérable ». Mais il aura fallu attendre cinq mois pour connaître cette sentence arbitrale. A quoi attribuer cette lenteur d'action? Au long temps pris pour nommer les arbitres? Ce qui est peu probable quant aux représentants du syndicat et de la compagnie, mais le choix du président du tribunal a pu être plus long surtout si les deux parties, faute de s'accorder, ont dû en laisser faire le choix par le ministre du Travail. L'explication la plus plausible peut-être, c'est que lorsque le tribunal fut prêt à siéger les vacances d'été l'en empêchèrent pratiquement. C'est pourquoi son rapport ne fut prêt qu'en octobre. Cinq mois, c'était tout de même exagéré pour disposer d'un unique grief relevant de l'application d'une clause de la convention collective.

La sentence arbitrale majoritaire, rendue le 20 octobre, reconnaissait à la Canadian Johns-Manville le droit d'effectuer le changement de tâche prévu, soit la diminution d'un homme sur une équipe donnée. Un point n'est pas clair ici: la compagnie n'effectua-t-elle ce changement qu'au lendemain de la sentence arbitrale. Le 20 octobre, ou l'avait-elle déjà mis en force depuis la fin de la conciliation mais sous protêt de la part du syndicat? *

Cette décision était-elle injuste envers le syndicat? Peut-être, partiellement, en n'ayant pas majoré quelque peu le salaire horaire

(4) M. OvILA CrR, président alors du syndicat, me dit lors d'une rencontre en 1962, que par un règlement à l'amiable entre la Compagnie et le Syndicat, le changement de tâche avait été effectué quelque temps avant que fut rendue la sentence arbitrale. 
de l'un des employés en cause auquel incombait un surcroit de responsabilité, et vu le gain de 0.80 cents de l'heure réalisé par la Compagnie depuis l'installation d'une pelle mécanique. D'autre part, la convention n'a pas paru pleinement violée due à l'absence de stipulation précise sur le changement de tâche, et les deux parties avaient accepté de se soumettre à la décision arbitrale.

Janvier 1949, reprise des négociations

Commencées en décembre 1948, les négociations se poursuivent, en janvier 1949, entre la « Fédération » et Canadian Johns-Manville, Asbestos Corporation et Johnson's Ltd.

Le 24 janvier 1949 la Fédération nationale des Employés de l'Industrie Minière Inc., informe la Commission de relations ouvrières de la rupture des négociations pour le renouvellement de la convention collective avec les trois compagnies précitées et demande l'intervention du Service de conciliation.

\section{Demande de conciliation}

Afin de donner suite à cette demande, la Commission s'enquiert aussitôt auprès de la Fédération des dates des rencontres avec les compagnies et des points sur lesquels il $\mathrm{y}$ a désaccord. Dès le lendemain, le 27 janvier, le secrétaire de la Fédération, M. Daniel Lessard, écrit à la Commission son désappointement d'avoir à fournir ces informations et il ajoute «nous ne voulons pas être dans la même situation qu'en février 1948. Enumérant ensuite les points sur lesquels il n'y a pas eu entente, M. Lessard presse la Commission de faire diligence avant que les ouvriers s'impatientent contre elle. Il dénonce particulièrement la mauvaise foi de la Canadian Johns-Manville qui a commencé d'afficher un bulletin pour informer les ouvriers sur la marche des négociations.

Sans tarder la Commission envoie au Service de conciliation tous les documents requis pour l'intervention conciliatrice. Elle en informe la Fédération et demande au sous-ministre du Travail de nommer un conciliateur. M. Gérard Tremblay demande, le 2 février, au Service de conciliation d'intervenir dans le différend « en exécution de l'article 13 de la Loi des relations ouvrières. Le conciliateur Léopold Rogers à qui cette tâche est assignée soumet un rapport préliminaire le 7 février au ministre du Travail.

\section{Rapport préliminaire du conciliateur}

Le conciliateur dit avoir assisté, le 31 janvier au soir, à l'assemblée des employés de la Johns-Manville à Asbestos et y avoir constaté les faits suivants: a) qu'il était question de voter la grève pour qu'elle commence à minuit le même soir; b) qu'il réussit à faire retarder ce vote de 24 heures, s'il obtenait, d'autre part, de la compagnie de cesser 
l'affichage de bulletins sur la marche des négociations; c) qu'il obtint de la compagnie de cesser tel affichage; d) qu'il poursuivit ses négociations les 2, 3 et 4 février très difficilement parce que la Fédération cherchait à standardiser tous les contrats à être renouvelés prochainement avec les autres compagnies minières de la région; e) que, de son côté, la Johns-Manville réclamait le droit de pouvoir adapter les clauses du contrat aux besoins de l'organisation de sa propre entreprise; f) que, de part et d'autre, toutefois, des clauses secondaires furent acceptées et quelques autres référées à l'étude de chaque parti; g) qu'enfin furent différées à plus tard les clauses relatives aux salaires, vacances, retenu de la cotisation syndicale et autres. Mais le conciliateur déclare en terminant qu'il appréhende, tôt ou tard, une épreuve de force entre les parties.

\section{Rapport final du conciliateur}

Ce qui précède est un rapport préliminaire du conciliatēur, il reprendra les séances de conciliation le 7 février jusqu'au 10 suivant inclusivement. Après quatre jours d'exténuants pourparlers, il restait de nombreux points sur lesquels l'entente était impossible. D'abord de la part du Syndicat, les demandes suivantes: l'inclusion dans la juridiction du syndicat des ouvriers employés par les sous-contracteurs; l'élimination des poussières d'amiante; l'application de la formule Rand; et autres demandes d'ordre monétaire; ensuite, de la part de la compagnie, deux demandes: la détermination des droits de la gérance et l'établissement d'une procédure pour l'étude des standards d’efficacité. $\mathrm{Vu}$ le grand nombre de sujets en suspens, les deux parties convinrent de soumettre tout le projet de convention à l'arbitrage. Mais le dimanche 13 février au soir, réunis en assemblée d'urgence, les ouvriers déclarèrent la grève effective à minuit.

A noter que le conciliateur soulignera dans son rapport au ministère du Travail que dans les séances de conciliation, la compagnie s'offusqua d'avoir à négocier avec la Fédération et s'objecta à sa tentative de vouloir uniformiser les conditions de travail dans l'industrie de l'amiante. Elle prétendait que les conditions de son exploitation étaient différentes de celles des compagnies concurrentes.

\section{LA GRève éclate $\dot{A}$ Asbestos}

Informé le lendemain de sa déclaration de grève depuis minuit à la Canadian Johns-Manville, le chef du service de conciliation télégraphie au président du syndicat, A. Larivée, le message suivant:

M. Armand Larivée

Montréal 14 février 1949

151 rue Notre-Dame

Asbestos.

Suis informé déclaration grève Canadian Johns-Manville Asbestos depuis hier soir stop attire votre attention sur violation article 24 Loi 
relations ouvrières stop Etant donné échec négociations et conciliation le ministère accorde tribunal d'arbitrage pour régler différend stop Vous devez donc recommander ouvriers de retourner au travail afin de permettre que procédure soit suivie et me faire parvenir en duplicata nom, prénom et adresse arbitre que vous désignerez stop Après retour au Travail tribunal d'arbitrage procédera sans délai.

\section{Miron, Directeur}

Service de conciliation et d'arbitrage.

Copie de ce télégramme est envoyée au président de la Fédération des mineurs, M. Rodolphe Hamel. Et M. Miron télégraphie à la Canadian Johns-Manville de nommer son arbitre.

\section{Déclaration de M. McGaw}

Dans la même journée du 14, M. Miron écrit au sous-ministre du Travail avoir appris du gérant des relations industrielles de la compagnie, M. McGaw, que les membres du Syndicat auraient accepté à l'assemblée de la veille, la recommandation des officiers d'aller à l'arbitrage, mais que c'est après l'assemblée qu'ils auraient décidé le recours à la grève. M. Miron appréhende que la grève sera aussi déclarée aux mines des compagnies «Johnson's » et «Asbestos Corporation » à l'assemblée que les employés doivent tenir mercredi soir. Le lendemain, la C.R.O. est informée des mêmes faits par le chef de Service de conciliation.

Télégramme de J. Marchand à l'Honorable A. Barrette

Faisant suite au télégramme de M. Miron, M. A. Larivée, M. Jean Marchand (devenu secrétaire général de la C.T.C.C.) répondit, par télégramme, le jour même, que l'avocat Théo. Lespérance sera arbitre du syndicat. Le texte authentique du télégramme de M. Marchand n'apparaît pas dans les dossiers de la C.R.O.; seulement nous le trouvons intercalé dans le long mesage télégraphique qu'envoya $\mathbf{M}$. Miron au sous-ministre du Travail le 14 février 1949 et que voici:

\section{CANADIAN PACIFIC TELEGRAPHS CO.}

\section{GERARD TREMBLAY}

FEBRUARY 11th, ${ }^{5}$ (14) 1949.

SOUS MINISTRE DU TRAVAIL HOTEL DU GOUVERNEMENT

QUEBEC, QUE.

TELEGRAMME SUIVANT REÇU DE JEAN MARCHAND SECRETAIRE GENERAL CTCC VOICI QUOTE COPIE DU TELEGRAM-

(5) La date du 11 février en tète du télégramme ci-dessus est une erreur de transcription, étant antérieure à la grève; le 14 doit être la date véritable dudit télégramme. 
ME ENVOYE CE JOUR A L'HONORABLE ANTONIO BARRETTE MINISTRE DU TRAVAIL RE DIFFERENDS CANADIAN JOHNS MANVILLE ASBESTOS CORPORATION LIMITED JOHNSONS COMPANY FLINTKOTE MINES LIMITED ET FEDERATION NATIONALE EMPLOYES DE L'INDUSTRIE MINIERE RECOMMANDE MAITRE LESPERANCE 1231 DEMONTIGNY EST MONTREAL COMME ARBITRE SYNDICAL SI VOUS CROYEZ DEVOIR INSTITUER TRIBUNAL DANS CES AFFAIRES STOP TELEGRAMME ENVOYE A JEAN MARCHAND QUOTE VOTRE TELEGRAMME REÇU RE CHOIX DE MAITRE THEO LESPERANCE PAR FEDERATION NATIONALE EMPLOYES DE L'INDUSTRIE MINIERE AGIR COMME MEMBRE TRIBUNAL ARBITRAGE DANS DIFFREND CANADIAN JOHNS MANVILLE ET SYNDICAT CE TRIBUNAL DOIT ETRE INSTITUE ETANT DONNE ECHEC CONCILIATION STOP VOUS MENTIONNEZ SUR TELEGRAMME TROIS AUTRES COMPAGNIES DOIS JE COMPRENDRE QUE MEME ARBITRE AGIRA POUR SYNDICAT DANS CHAQUE CAS SI NOUS DEVONS INSTITUER TRIBUNAL DANS DIFFERENDS CAS STOP POUR LE MOMENT MAITRE LESPERANCE EST CONSIDERE ARBITRE SYNDICAL CAS CANADIAN JOHNS MANVILLE STOP PRIERE DE FAIRE PARVENIR EN DUPLICATA TELLE NOMINATION SUR DOCUMENT OFFICIEL STOP VOUS N'IGNOREZ PAS QUE OUVRIERS ONT DECLARE GREVE EN DEPIT DU FAIT QUE LA PROCEDURE D'ARBITRAGE N'A PAS ETE SUIVIE STOP APRES LECTURE DE CES TELEGRAMMES MONSIEUR TREMBLAY VOUS VOUS RENDREZ COMPTE DE L'INTENTION DE JEAN MARCHAND DE TENTER D'AMENER CAS DES QUATRE DIFFERENTES COMPAGNIES DEVANT MEME TRIBUNAL D'ARBITRAGE STOP SUIS CONVAINCU QUE DIFFERENTES COMPAGNIES N'ACCEPTERONT PAS CETTE PROCEDURE LA EST LA RAISON DE MA REPONSE PRIERE M'INFORMER PROCEDURE QUI SERA SUIVIE PAR MINISTERE.

\section{MIRON}

Le secrétaire de la C.T.C.C., Jean Marchand, demande au ministère du Travail d'instituer un conseil d'arbitrage non seulement pour les trois compagnies de Thetford-Mines où la conciliation vient d'échouer, mais qui disposerait également du différend de la Canadian JohnsManville déjà en grève. Et ignorant, apparemment à dessein, ce fait le secrétaire de la C.T.C.C. recommande M. Théo Lespérance comme arbitre syndical pour les quatre différends, si le ministre «croit devoir former un tribunal en cette affaire ». Soit donc un seul tribunal!

L'unique tribunal d'arbitrage demandé par Jean Marchand pour quatre entreprises différentes - bien que dans la même industrie était une procédure contraire à l'interprétation constante donnée jusque là à la Loi des relations ouvrières pour la C.R.O., à savoir que ladite loi prescrit linstitution d'un conseil d'arbitrage dans le cas de toute 
entreprise distincte soumise à un différend et qui a épuisé le stade de la conciliation. Le chef du Service de conciliation avant raison de craindre l'opposition des compagnies à cette procédure.

\section{La grève s'étend d trois autres mines}

Au moment où éclata la grève à la Canadian Johns-Manville une autre grève était en cours depuis deux semaines à la Nicolet Asbestos Mines de St-Rémi de Tingwick, puis pendant la journée du 14 février où se fait l'échange des télégrammes précités entre Jean Marchand et le Chef du Service de conciliation la grève s'étend aux mines des trois firmes Asbestos Corporation Ltd., Johnson's Company et Flintkote Mines Ltd. La conciliation en cours, depuis le 11 février, entre ces trois compagnies et leurs syndicats respectifs pour renouveler leurs conventions avait atteint un point mort, ainsi qu'en témoigne le télégramme ci-après que le directeur du Service de conciliation adressa à M. Gérard Tremblay le 15 février:

CANADIAN NATIONAL TELEGRAPHS

Montréal, 15 février 1949

Monsieur Gérard Tremblay

Sous-Ministre du Travail

Hôtel du Gouvernement

Québec, Qué.

VOUS ETES INFORME SUREMENT DU FAIT QUE OUVRIERS TROIS COMPAGNIES ASBESTOS CORPORATION JOHNSONS COMPANY FLINTKOTE MINES LTD ONT DECLARE GREVE HIER SOIR OU CE MATIN STOP DOIS VOUS DIRE QUE CONCILIATEUR ROGERS A TENU DERNIERE SEANCE HIER APRES MIDI ET IL A ETE ENTENDU AVEC OUVRIERS NEGOCIATEURS SYNDICATS QUE DIFFERENDS ETAIENT REFERES ARBITRAGE STOP MONSIEUR ROGERS ET OFFICIERS SYNDICATS ONT LAISSE THETFORD MINES POUR SE RENDRE ST REMI DE TINGWICK ET SE SONT RENDUS COMPTE A LEUR RETOUR A THETFORD QUE GREVE ETAIT DECLAREE STOP J'AI RETELEGRAMME SUIVANT AUX TROIS PRESIDENTS DES TROIS SYNDICATS AINSI QU'AU SECRETAIRE FEDERATION DANIEL LESSARD QUOTE SUIS INFORME DECLARATION GREVE DEPUIS HIER MATIN STOP ATTIRE VOTRE ATTENTION SUR VIOLATION ARTICLE VINGT QUATRE LOI RELATIONS OUVRIERES STOP ETANT DONNE ECHEC NEGOCIATION ET CONCILIATION LE MINISTERE ACCORDE TRIBUNAL D'ARBITRAGE POUR REGLER DIFFEREND STOP VOUS DEVEZ DONC RECOMMANDER OUVRIERS DE RETOURNER AU TRAVAIL AFIN PERMETTRE QUE PROCEDURE SOIT SUIVIE ET DE FAIRE PARVENIR EN DUPLICATA NOM PRENOMS ET 
ADRESSE DE L'ARBITRE QUE VOUS DESIGNEZ STOP APRES RETOUR AU TRAVAIL TRIBUNAL D'ARBITRAGE PROCEDERA SANS DELAI FIN CITATION.

\section{Cyprien Miron, directeur}

Service de conciliation et d'arbitrage

Au soir du 14 février, se réunissant d'urgence, les employés des trois compagnies minières de Thetford Mines décident de se joindre aux grévistes d'Asbestos. Ces derniers qui avaient, dans chaque cas, épuisé le stade de la conciliation passèrent outre à celui de l'arbitrage; ils débrayèrent spontanément en l'absence du président et du secrétaire de la Fédération, avant leur retour de St-Rémi de Tingwick.

Télégramme du ministre du travail d la C.R.O.

Deux jours après l'envoi du télégramme du secrétaire de la C.T.C.C. au ministre du Travail demandant d'instituer un unique conseil d'arbitrage, l'hon. Barrette lui télégraphia, le 17 février, le message suivant reproduit d'après la copie dudit message envoyé le même jour au Juge Conrad Pelletier. ${ }^{\circ}$

\section{CANADIAN NATIONAL TELEGRAPHS}

QUEBEC QUE. 17

HON. JUGE CONRAD PELLETIER, PRESIDENT COMMISSION DES RELATIONS OUVRIERES 7080 HUTCHISON ST MTL

TELEGRAMME SUIVANT A ETE ENVOYE JEAN MARCHAND HAMEL ET MIRON 15 FEVRIER QUOTE SOMMES INFORMES OFFICIELLEMENT PAR MONSIEUR CYPRIEN MIRON DIRECTEUR SERVICE CONCILIATION ET ARBITRAGE DE LA GREVE DES EMPLOYES DE LA CANADIAN JOHNS-MANVILLE ASBESTOS CORPORATION LIMITED JOHNSON COMPANY FLINTKOTES MINES LIMITED FEDERATION NATIONAL DES EMPLOYES DE L'INDUSTRIE MINIERE STOP NOTONS QUE LES PARTIES N'ONT PAS RECOURU A PROCEDURES REGULIERES STOP CEPENDANT RECEVONS AVIS SOUS SIGNATURE DE MONSIEUR JEAN MARCHAND EN DATE DU 14 QUE LA FEDERATION NATIONALE DES EMPLOYES DE L'INDUSTRIE MINIERE RECOMMANDE MAITRE T.O. LESPERANCE COMME REPRESENTANT SYNDICAL SI MINISTERE CROIT DEVOIR INSTITUER TRIBUNAL D'ARBITRAGE STOP.

(6) Le dossier de la C.R.O. particulier à la Canadian Johns-Manville ne contient pas le texte authentique du télégramme qu'envoya l'honorable Barrette à Jean Marchand. 
1-NOUS NE POUVONS QUE CONDAMNER UNE GREVE GENERALE FAITE EN VIOLATION FORMELLE DE L'ARTICLE 24 PARAGRAPHE 1 DE LOI DES RELATIONS OUVRIERES STOP.

2-NOUS SOMMES DISPOSES A FORMER UN TRIBUNAL D'ARBITRAGE CE TRIBUNAL NE SERA CONSTITUE QUE LORSQUE LES EMPLOYES SERONT RENTRES DANS LA LEGALITE EN RETOURNANT AU TRAVAIL STOP.

3-NOUS SERONS DE PLUS DANS LA NECESSITE SI LA SITUATION SE CONTINUE D'AVISER COMMISSION RELATIONS OUVRIERES DE L'ILLEGALITE DE CETTE GREVE L'INVITANT A CONSIDERER LE RETRAIT DE LA CERTIFICATION STOP.

4 -VOUS RECOMMANDONS DONC D'INVITER LES OUVRIERS A SE MONTRER RESPECTUEUX DES LOIS DANS LEUR PROPRE INTERET ET CELUI DE LEUR CAUSE EN CESSANT LA GREVE ET EN CONFIANT A UN TRIBUNAL D'ARBITRAGE LE SOIN DE RENDRE JUSTICE AUX DEUX PARTIES UNQUOTE. POURREZ RETIRER CERTIFICATION SI JUGEZ A PROPOS.

Antonio Barrette, Ministre du Travail

Avertissement de la C.R.O.

Le même jour (17) que l'hon. Barrette envoie le télégramme plus haut cité à la C.R.O. celle-ci, à son tour, de la même façon, adresse le même avertissement à la Fédération ainsi qu'à ses syndicats de Thetford-Mines et d'Asbestos.

Les grévistes passant outre à cet avertissement, la Commission répète son ordonnance deux jours après en édictant qu'à défaut de leur retour du travail au plus tard le 21 février les certificats de reconnaissance des syndicats en cause * seront révoqués 》.

\section{Protestation de la fédération}

Dès le lendemain, la Fédération proteste contre cette ordonnance par le télégramme suivant:

Montréal, 20 février, 1949

Monsieur P.-E. Bernier, secrétaire,

Commission de relations ouvrières,

286 rue St-Joseph

Québec, Qué.

VOTRE TELEGRAMME DU DIX NEUF REÇU STOP FEDERATION NATIONALE DES EMPLOYES INDUSTRIE MINIERE EN SON NOM ET AU NOM DES SYNDICATS CONCERNES PROTESTE CONTRE MENACE DE RETIRER CERTIFICAT RECONNAIS- 
SANCE SYNDICALE STOP CONSIDERE CETTE MESURE NON JUSTIFIEE PAR LOI RELATIONS OUVRIERES NI PAR LES FAITS ET DEMANDE AUDITION AVANT TOUTE DECISION.

Daniel Lessard, Secrétaire Fédération Nationale des employés de l'Industrie Minière.

Révocation des certificats

Le jour même (21 février) qu'elle reçoit le télégramme ci-dessus la C.R.O. refuse d'entendre la protestation de la Fédération au nom des syndicats, parce que ceux-ci ont ignoré sa mise en demeure, et elle décide de révoquer leurs certificats de reconnaissance. Décision transmis par télégramme le lendemain et à la Fédération et aux syndicats.

Dans le cas particulier de la Canadian Johns-Manville voici le texte de la décision de la C.R.O.:

\section{COMMISSION DE RELATIONS OUVRIERES}

\section{DECISION}

Syndicat de l'Amiante d'Asbestos Inc., Asbestos, P.Q.

Canadian Johns-Manville Co. Ltd Asbestos, Qué.

ATTENDU le certificat de reconnaissance syndicale émis, le 29 octobre 1947, en faveur du SYNDICAT NATIONAL DE L'AMIANTE D'ASBESTOS INC., ET DONT l'agent négociateur est la Fédération nationale des Employés de l'Industrie minière Inc., dûment mandatée par le Syndicat, pour représenter «tous les employés rémunérés sur une base horaire, à la pièce ou à la tâche, à l'exception des contremaîtres, employés de bureau, employés de moins de 16 ans », à l'emploi de CANADIAN JOHNS-MANVILLE CO. LTD, ASBESTOS;

ATTENDU la grève actuellement en cours parmi les salariés de Canadian Johns-Manville Co. Ltd, Asbestos;

ATTENDU la mise en demeure adressée aux parties syndicales intéressées le 17 courant et complétée le 19 février d'avoir à rentrer au travail au plus tard lundi le 21 février 1949 sous peine des sanctions prévues et de la perte de leurs droits selon les dispositions de la Loi des relations ouvrières de la province de Québec;

CONSIDERANT que les parties syndicales intéressées n'ont pas donné suite à cette mise en demeure; 
CONSIDERANT que cette grève est illégale au sens du paragraphe 1 de l'article 24 de la Loi des relations ouvrières;

CONSIDERANT les dispositions de la Loi des relations ouvrières de la Province de Québec et tout particulièrement celles de l'article 21 ;

\section{POUR CES MOTIFS, la Commission DECIDE:}

DE REVOQUER à toutes fins que de droit le certificat de reconnaissance syndicale émis le 29 octobre 1947 en aveur du SYNDICAT NATIONAL DE L'AMIANTE D'ASBESTOS INC., et dont l'agent négociateur est la Fédération Nationale des Employés de l'Industrie Minière Inc., dûment mandatée par le Syndicat, pour représenter « tous les employés rémunérés sur une base horaire, à la pièce ou à la tâche, à l'exception des contremaîtres, employés de bureau, employés de moins de 16 ans ». à l'emploi de Canadian Johns-Manville Co. Ltd., Asbestos; le tout conformément aux dispositions de la Loi des relations ouvrières de la Province de Québec.

QUEBEC ce 2lième jour de février 1949

Le président: Juge L.-C. Pelletier

Vice-Président: H.-C. Lebrun

Les Commissaires: Candide Rochefort

P.-E. Brais

J. Gosselin

Menace de décertification - Offre de compromis

Offre d'arbitrage spéciale

Par son télégramme du 17 février, le ministre du Travail répond à Jean Marchand qu'il condamne la grève générale, parce qu'elle est illégale; qu'il instituera un tribunal d'arbitrage après le retour des grévistes au travail; qu'il avisera la C.R.O. de décertifier les syndicats si l'illégalité persiste.

Placé en face d'une grève qui concerne quatre compagnies le conseil d'arbitrage, que l'hon. Barrette est disposé à instituer conditionnellement, semble devoir être un tribunal unique pour les quatre différends. Si tel étaient ses dispositions le ministre s'écartait de la procédure habituelle. Par un tribunal d'arbitrage unique les syndicats obtenaient un moyen important pour les aider à uniformiser leurs conditions de travail, d'autant plus que leur agent négociateur allait être leur Fédération déjà reconnue comme tel par la C.R.O. N'eut-il pas été sage alors d'accepter le retour au travail? Cependant la menace de décertification des syndicats par le ministre du Travail était sûrement un abus de pouvoir en même temps qu'une violation des prescriptions de la Loi. 
Lorsqu'à son tour la C.R.O. menaça deux fois, les 17 et 19 février, de révoquer les certificats, elle aussi excédait ses pouvoirs. Elle commettait ensuite un déni de justice en refusant d'entendre en séance d'audition la protestation de la Fédération. Elle commettait enfin un faux contre la Loi, le 21 février, en révoquant les certificats de reconnaissance syndicale.

Du lendemain de la révocation des certificats et jusqu'au 10 mars, les évènements qui s'intercalent sont les suivants selon les rapports des journaux: a) arrivée d'un détachement de la police provinciale à Asbestos; b) demande d'une entrevue entre les représentants du syndicat et le ministre du Travail de même qu'avec le Premier ministre mais, entrevue que les représentants ouvriers refusent d'avoir sans être accompagnés par Jean Marchand, que veut évincer M. Duplessis; c) le même jour, en chambre, le Premier ministre traite de «saboteurs 》 des lois les chefs de la grève; d) la mine «Bell » annonce qu'elle ferme sa mine le samedi suivant.

\section{Offre de médiation du ministre du travail}

Vers le 10 mars, le ministre du Travail offre sa médiation, si le retour au travail est ordonné aux grévistes. Il déclare qu'advenant le retour au travail, il recommandera la recertification des syndicats.

\section{Echec des négociations à East-Broughton}

Le 22 mars marque l'échec des négociations à la Quebec Asbestos Corporation de East-Broughton. Aussitôt le secrétaire de la C.T.C.C. télégraphie à la C.R.O. pour lui demander l'intervention conciliatrice. Le principal point en litige est la sécurité syndicale selon la «formule Rand ». (A quelques jours de là le ministre du Travail s'oppose publiquement à la «formule Rand», la croyant illégale, et il s'ensuit une polémique entre lui et le secrétaire général de la C.T.C.C.) Le 6 avril, le conciliateur Noël Bérubé rapporte que la conciliation s'avère infructueuse et que tout le projet de convention soumis par le syndicat doit être référé à un conseil d'arbitrage.

Ce conseil est bientôt formé du Juge Joseph Bilodeau et des avocats Paul Smith et Théo Lespérance. Mais, en juillet, il devra obtenir du ministre du Travail, la permission de différer jusqu'en septembre la date où il doit faire son rapport. Le dit rapport est effectivement terminé le 23 août 1949. Et il contient un projet de convention collective dont s'inspireront les parties lorsqu'elles signeront leur convention le 10 novembre.

\section{Compromis refusé par C.J.M.}

Par ailleurs, durant le mois d'avril, la grève continuait de se poursuivre à Asbestos ainsi qu'à Thetford-Mines. Les journaux rapportèrent les faits suivants durant ce mois: 
Vers la mi-avril des rencontres sont amorcées entre le représentant des syndicats et ceux des quatre compagnies dont les opérations sont suspendues. Les trois compagnies de Thetford-Mines: Asbestos Corporation, Johnson's Ltd, et Flintkote Co., considéraient la possibilité d'un compromis, en donnant des garanties écrites qui permettraient aux grévistes de retourner au travail sans crainte de représailles. Mais de son côté Canadian Johns-Manville d'Asbestos résista à cette offre de compromis par l'envoi, au contraire, de lettres aux grévistes les appelant au travail et par la tentative de tenir à ce sujet un référendum parmi eux. La lettre les avertissait en outre qu'ils risquaient de perdre leur emploi ainsi que leurs bénéfices sociaux s'ils ne retournaient pas au travail.

\section{Barrette propose un arbitre unique}

Vers le 10 avril, l'hon. Barrette offre sa médiation aux grévistes mais vainement. Le 16 il leur fait une nouvelle proposition. Il leur promet, conditionnellement à leur retour au travail, de nommer tout de suite un juge comme arbitre unique pour entendre leur différend et que ce juge rendrait une décision expéditive. Après, les grévistes resteraient libres de reprendre la grève s'ils le voulaient, cette fois légalement.

Cette proposition du ministre du Travail fut annoncée dans le temps par le journal le «Devoir», lequel disait que M. Gérard Picard devait l'étudier le soir même à l'assemblée des grévistes à Asbestos. Mais le «Devoir» ne publia pas de compte rendu de cette assemblée. Apparemment la proposition fut rejetée ou l'on adopta une contre-proposition que l'on a transmise sans publicité au ministre du Travail. Il est certain que la proposition Barrette ne pouvait être acceptée sans garanties écrites quaucune représaille ne serait exercée contre aucun gréviste par les compagnies.

\section{Causerie radiodiffusée de l'Honorable Barrette}

Peu de jours après, l'Hon. Barrette prononce une importante causerie radiodiffusée. Rapportée par les journaux, voici un résumé de la causerie de M. Barrette: il tente de faire le point dans le conflit de l'amiante. Après avoir relaté l'origine de la grève et les faits qui l'ont suivi il rappelle l'offre de médiation qu'il fit le 9 mars et déclare que le président de la Fédération, M. Rodolphe Hamel, lui avait déclaré que son offre présentait des garanties suffisantes. Il ajoute par contre qu'une délégation du conseil municipal d'Asbestos l'a informé qu'effectivement son offre antérieure était acceptée par les grévistes, mais qu'ils exigeaient que leurs principales demandes fussent acceptées sans discussion. L'Hon. Barrette conclua que devant pareille condition il ne pouvait plus agir comme arbitre impartial.

Le lendemain de sa causerie les grévistes demandèrent la démission de l'Hon. Barrette. 


\section{LE REेGLEMENT}

Pourparlers chez le ministre du travail

Durant la dernière semaine d'avril des pourparlers s'engagèrent en présence du ministre du Travail entre, d'une part M. Yvan Sabourin et, d'autre part, Me Théo. Lespérance accompagné de M. Gérard Picard. Ces conversations n'ayant rien donné, elles furent rompues le 30 avril. Restait le recours à l'arbitrage, mais avant de recourir à ce moyen la Canadian Johns-Manville proposa, le 29, une formule de règlement de la grève en trois points, et qui se lisait comme il suit:

Entente relative au retour au travail des grévistes de l'industrie de l'amiante.

1-Dès la fin de la grève, le ministre du Travail recommandera à la Commission des Relations ouvrières que le certificat de reconnaissance syndicale soit remis au Syndicat dont les membres sont en grève et cela tel qu'il existait auparavant. La compagnie ne s'objecte pas à telle remise du certificat.

2-Aucune discrimination du fait de la grève ne sera exercée contre aucun employé et ceux-ci seront remis à leur travail le plus rapidement possible et d'accord avec les besoins de la production.

3-Le ministre du Travail est disposé à faire toutes démarches utiles auprès de la compagnie en vue d'amener un règlement à l'amiable des procédures civiles perdantes.

\section{Exigences syndicales}

S'opposant à ces offres dans le bureau du ministre du Travail les syndicats présentèrent les exigences suivantes:

1-Dès la fin de la grève, le certificat de reconnaissance syndicale sera remis au syndicat dont les membres sont en grève, et cela tel qu'il existait auparavant, aucune objection de la part de la compagnie concernée ne sera soulevée contre la recertification.

2-Aucune discrimination du fait de la grève ou à la raison d'incidents relatifs à la grève ne sera exercée contre aucun employé, et tous les employés seront remis au travail dans un délai maximum de cinq jours, à moins de raison incontrôlable, et ils auront la préférence pour l'emploi qu"ils occupaient avant la grève.

3-Les actions et injonctions intentées contre certains organismes syndicaux (C.T.C.C., Fédération de l'industrie minière et syndicats locaux) seront considérées réglées et nulle autre action relative à la grève sera intentée contre lesdits organismes syndicaux, leurs officiers ou leurs membres.

Comment se comparent ces propositions? Que s'en dégage-t-il? Premièrement, le refus de la compagnie d'accepter les exigences du syndicat relativement à la non-discrimination, décelait son intention de maintenir certaines procédures civiles soit contre des grévistes, soit con- 
tre des officiers ou le Syndicat. Deuxièmement, rien ne garantissait dans les offres de la Canadian Johns-Manville que le ministre du Travail eut pu obtenir de la compagnie les ménagements désirés en faveur des grévistes pour faciliter leur retour au travail ainsi que la reprise des négociations.

Le 3 mai, la Canadian Johns-Manville exhorte les mineurs par les journaux à retourner au travail. Un petit nombre rentre au travail, rejoint par quelques ouvriers étrangers. Le lendemain la compagnie se dit prête à négocier avec les représentants du syndicat local d'Asbestos. Ce qui était tenter de dissocier ce dernier de la Fédération.

\section{Formation d'un conseil d'arbitrage}

Les deux parties étant restées sur leurs positions, chacune, le 5 mai, nomme son représentant sur un tribunal d'arbitrage: M. Yvan Sabourin pour la compagnie et Me Théo Lespérance pour le Syndicat. Lesquels, après quelques jours, n'ayant pu s'entendre pour choisir le président du tribunal, demandent au ministre du Travail de le nommer.

Rappelons ici que le 5 mai fut aussi la journée où une émeute éclata à Asbestos entre grévistes et policiers provinciaux.

Propositions de règlement de grève

Le 17 mai, le susdit tribunal n'étant apparemment pas encore nommé, la « Johns-Manville» envoie au ministre du Travail une nouvelle proposition de règlement de grève. Cette proposition ayant été soumise par le ministre au Syndicat, ce dernier lui adresse une contre-proposition. Peu de jours après, convoquées dans le bureau du ministre du Travail, les deux parties prennent connaissance d'une proposition de compromis offerte par l'Hon. Barrette. Des négociations s'ensuivent dans le bureau du ministre pendant une semaine. Mais les parties ne peuvent s'entendre surtout sur les conditions de retour au travail. Le Syndicat s'opposa à ces dernières à cause de leurs restrictions contre certains grévistes. Le syndicat posait une autre difficulté non moins grave à surmonter; il demandait qu'advenant l'échec de la reprise des négociations pour une convention collective, soit institué un conseil d'arbitrage sans passer par le stade de la conciliation.

Bien que la loi de relations ouvrières prescrive qu'il faille d'abord chercher à concilier avant d'arbitrer un différend qui découle de négociations infructueuses, justifiée était l'exigence du syndicat de supprimer le stade de la conciliation car ce stade avait été expérimenté. Une dernière exigence du syndicat était que le président du conseil d'arbitrage soit le juge en chef de la Cour d'appel ou un autre nommé par lui. Légalement, pareille nomination relève exclusivement du ministre du Travail quand les parties en litige ne s'entendent pas pour faire ce choix. Cela montra l'extrême méfiance des représentants des grévistes contre le gouvernement. Preuve que ces derniers redoutaient fortement la collusion qui avait paru exister entre le gouvernement et la Canadian 
Johns-Manville dès le début du conflit. Puis, l'Hon. Barrette avait soumis une proposition de compromis qu'accepta le représentant de la compagnie, mais que refusa partiellement Gérard Picard au nom du syndicat.

\section{Position des syndicats}

Des huit points de la proposition Barrette, Gérard Picard s'objecta aux clauses 2,3 et 7 . La clause 2 assurait le retour au travail des grévistes mais ne garantissait pas qu'aucune discrimination ne serait exercée contre aucun d'eux. La clause 3 réservait à la compagnie le droit de suspendre tout employé sous le coup d'une accusation criminelle ainsi que le droit de refuser de reprendre ceux qui seraient trouvés coupables. La clause 7 garantissait après le retour au travail, la reprise des négociations pour une convention collective et, si nécessaire, le recours à la conciliation et à l'arbitrage selon la Loi.

Echec de la 2ème tentative de règler la grève

C'est donc le 2 juin, dans le bureau du ministre du Travail, qu'échoua la deuxième tentative du règlement de la grève. Le syndicat s'en tenait à sa proposition du 24 mai. Au désaccord entre les parties sur les conditions de retour au travail, s'ajouta le désaccord persistant entre le syndicat et le ministre du Travail sur une procédure d'arbitrage en marge de la Loi qu'exigeait le Syndicat.

\section{Reglement de la grève en trois étapes}

En juin l'on s'acheminera graduellement vers le règlement final de la grève générale. Le 8 , s'effectue un règlement à la Nicolet Mines de St-Rémi de Tíngwick.

Le 18, les grévistes d'Asbestos sont appelés à voter sur une proposition de retour au travail faite par la Canadian Johns-Manville. Par un vote majoritaire de 95.7 pour cent des 1100 membres présents le Syndicat rejette l'offre de la compagnie, parce qu'elle ne protège pas les grévistes contre toutes représailles et que la procédure d'arbitrage ne leur convient pas.

Le 24, à Thetford-Mines, s'effectue un règlement conjoint entre les trois syndicats de cette ville et les compagnies « Asbestos Corporation 》, «Flintkote Mine» et «Johnson's». Par cette entente les trois compagnies précitées acceptent: a) la recertification des trois syndicats; b) le replacement des employés dans leurs occupations régulières le plus vite possible; c) la non-discrimination contre aucun employé due à la grève, sauf peut-être, contre ceux qui seraient trouvés coupables d'offenses graves; d) la reprise des négociations d'après les termes de la convention de 1949 , et, après dix jours, s'il n'y a pas entente, la nomination du Juge Thomas Tremblay, à moins quil $\mathrm{y}$ ait entente sur un autre choix. 
Le gouvernement consent, par exception, à ce qu'un seul conseil d'arbitrage dispose des trois causes à la fois. Cependant les compagnies de Thetford Mines consentent que l'arbitrage ne porte que sur les cas de *Asbestos Corporation 》, «Johnson's > et \& Flintkote Mine 》; mais cette dernière compagnie déclare se rallier à la sentence arbitrale touchant les deux précédentes compagnies. ${ }^{\text {? }}$

Enfin le 27 juin, survient le règlement final de la grève à la JohnsManville d'Asbestos. Cette entente, signée dans le même esprit que la précédente, est un peu plus élaborée en raison de l'envergure de l'entreprise et due à la particulière gravité du conflit à la même entreprise. Notons surtout les points suivants de cette entente, à savoir: la compagnie s'engage dे effectuer avec le ministre du Travail un règlement à l'amiable des procédures judiciaires et à étudier un plan de sécurité industrielle pendant la durée de la convention collective. D'autre part la compagnie obtient que soit insérée dans la convention une clause concernant les droits de la gérance. Enfin, il est stipulé que les employés retourneront au travail aux taux de salaires actuellement en vigueur.

\section{Recertification de syndicats}

Par suite des ententes mettant fin à la grève de l'amiante à Thetford Mines ainsi qu'à Asbestos, le ministre du Travail recommanda à la C.R.O. de remettre leurs certificats de reconnaissance aux quatre syndicats concernés. La Commission crût d'abord devoir exiger des syndicats, de soumettre une nouvelle requête en reconnaissance syndicale, mais se ravisant au bout de quelques jours, elle arrêta une procédure uniforme à cet effet. Le 13 juillet elle rescinda simplement sa décision de révocation des certificats en date du 21 février 1949.

Le tribunal d'arbitrage dans Asbestos Corporation rendit sa sentence le 10 décembre 1949. Les syndicats de Thetford non contents de certaines clauses, s'opposèrent particulièrement à la recommandation de signer une convention collective pour deux années, ce qui venait en contravention à la Loi des relations ouvrières. Il s'ensuivit des négociations post-arbitrales. La convention fut signée le 8 janvier 1950.

A la Canadian Johns-Manville la convention collective sera signée le 21 février 1950.

\section{ANNEXE}

\section{RAPPORT DU CONCILIATEUR LEOPOLD ROGERS}

Le conciliateur Léopold Rogers commence ses démarches le 11 février 1949 pour les terminer le 14 suivant. Pendant cette période il tient trois séances, fait

(7) Le 3 juillet ce tribunal est composé des avocats Théo. Lespérance pour le syndicat, Raymond Caron pour la compagnie et du Juge Thomas Tremblay, comme président. Le rapport du tribunal sera suivi d'une convention collective, signée le 13 janvier 1950. 
11 visites et a 5 entrevues. Au début de son rapport Monsieur Rogers déclare que le 14 février il a tenu deux séances de conciliation conjointe avec les représentants des deux compagnies « Asbestos Corporation * et « Johnson's 》 au bureau de la première, et son rapport se continue comme suit textuellement:

c A la séance de l'avant-midi, les parties ont repris pour le bénéfice des représentants de la compagnie \& Johnson's > la discussion sur la demande syndicale à l'effet de payer le temps supplémentaire aux employés travaillant à la pièce sur la moyenne horaire de leurs gains hebdomadaires. De nouveau, tout l'avant-midi y a passé, dans la discussion et l'expression du point de vue de chacune des parties, concernant ce point. En vain, le conciliateur a tenté d'accélérer le progrès des négociations.

\& La reprise des négociations à la séance de l'après-midi apporta l'inévitable. Alors que M. Penhale, président de \& Asbestos Corporation \$, eut exposé brièvement la situation sur le résultat et le progrès des négociations, au stade de la conciliation, sollicita du conciliateur, la recommandation au ministre du Travail de former un Conseil d'arbitrage pour régler le différend.

- A plusieurs occasions, le conciliateur a tenté de provoquer chez les parties, certains compromis, ce fut inutile et il se buta à un refus des deux côtés. Evidemment on est ouvert aux compromis possible, mais personne ne veut définitivement faire les premiers pas. Alors, où il fallait attendre, ou donner suite à la requête patronale et demander la formation d'un Conseil d'Arbitrage. Il fallut donc convenir que la formation d'un Conseil d'arbitrage dans le plus bref délai possible pourrait, peut-être, agréer aux ouvriers et apporter au litige une solution acceptable sinon satisfaisante dans les circonstances. Les parties acceptèrent de nommer leur arbitre pour la journée et faire parvenir leur nom au Service de conciliation et d'arbitrage.

- Aussitôt que la séance fut levée, le conciliateur tenta sans succès une dernière fois de convaincre les représentants patronaux à faire les premiers pas vers un compromis possible. Le même résultat négatif fut obtenu chez les représentants syndicaux.

e Plus tard et pendant que le conciliateur, accompagné du président du Syndicat, M. Géo. Dionne, du président et du secrétaire de la Fédération, MM. R. Hamel et D. Lessard, se rendaient auprès des grévistes de la Nicolet Asbestos Corporation à St-Rémi de Tingwick, les mineurs de la région de Thetford-lesMines prenaient sur eux d'organiser une assemblée d'urgence ${ }^{8}$ et insistèrent auprès des autres officiers négociateurs demeurés à Thetford-les-Mines, de leur donner un compte-rendu du résultat des négociations à date.

\& Le lendemain, au retour de St-Rémi de Tingwick à Thetford-les-Mines, le conciliateur et ses compagnons constatèrent, non sans étonnement, que plusieurs plants d'amiante avaient cessé leurs opérations et que les mineurs avaient dû déclarer la grève durant leur absence. Il était alors 5.00 a.m. Nous avions dû travailler toute la nuit pour régler la grève de St-Rémi pour constater à notre retour à Thetford-les-Mines que linévitable dans le conflit actuel venait de se produire.

- Cette grève, conséquence d'une longue préparation syndicale, devait nécessairement arriver et malgré les efforts constants du conciliateur à trouver une solution au présent litige. Le conciliateur n'a réussi qu'à la retarder temporairement.

Le 19 février 1949.

Signó: LEOPOLD ROGERS

(8) Les soulignés sont de l'auteur de l'artacle. 